\title{
Bowel preparation in colorectal surgery: back to the future?
}

\author{
Alice Frontali ${ }^{1} \cdot$ Yves Panis $^{1}{ }^{1}$ \\ Received: 5 June 2019 / Accepted: 7 June 2019 / Published online: 14 June 2019 \\ (C) Italian Society of Surgery (SIC) 2019
}

Mechanical bowel preparation (MBP) for colorectal surgery aims to reduce fecal mass and bacterial count with the objective to decrease surgical site infections (SSI) rate, including anastomotic leakage [1].

Many randomized studies (RCT) and meta-analysis [2, 3] comparing MBP versus no MBP have been published to date in colorectal surgery. No benefit of MBP in terms of SSI rate or anastomotic leakage has been suggested in all of these studies which mainly included patients operated for colonic surgery. We performed the only randomized study dedicated to rectal cancer surgery, the Greccar III [4] trial, which on the opposite demonstrated that after sphincter-saving rectal resection, 30-day overall (27\% with MBP vs $44 \%$ without; $p=0.018)$ and infectious ( $16 \%$ vs $34 \% ; p=0.005)$ morbidity rates were significantly lower in MBP versus no MBP group.

Thus, to date, MBP is recommended only for rectal cancer surgery and not for patients undergoing colonic surgery.

In the 1970s, some studies [5, 6] on the association of MBP and oral antibiotic (OA) have been appearing in literature and already at that time, it had been demonstrated that $\mathrm{MBP}+\mathrm{OA}$ was associated with reduced rates of SSI compared with MBP alone. Already in 1973, Nichols et al. [5] wrote that "elective colon resection should be approached with adequate preoperative mechanical and oral antibiotic preparation". However, these old studies have not convinced colorectal surgeons. And after these old papers, all the RCT have evaluated only MBP alone versus no MBP in colorectal surgery.

However, since these RCT and meta-analysis compare only MBP vs no MBP, recent large national surveys, including a huge number of patients from large databases, coming especially for US have suggested the possible benefits of oral

Yves Panis

yves.panis@aphp.fr

1 Department of Colorectal Surgery, Pôle des Maladies de l'Appareil Digestif (PMAD), Beaujon Hospital, Assistance Publique-Hôpitaux de Paris (AP-HP), University Denis Diderot (Paris VII), 100 Boulevard du Général Leclerc, 92110 Clichy, France antibiotics (OA) before colorectal surgery with a significant impact on SSI rate after colorectal surgery.

For this reason, in the last American 2019 clinical practice guidelines [7] concerning MBP for elective colorectal surgery, it is proposed that MBP combined with preoperative OA is typically recommended for elective colorectal resections with a strong grade of recommendation, based on moderate-quality evidence (grade 1B). Although this statement is mainly based on retrospective studies, the impact of OA seems very important in terms of reduction of SSI.

Ohman et al. [8] analyzed patients from the American College of Surgeons NSQIP and observed among their patients that those who received a full bowel preparation with both OA and MBP, a SSI rate of $2.7 \%$ was observed versus $15.8 \%$ without $(p<0.001)$. On multivariate analysis, full bowel preparation was independently associated with significantly fewer SSI (adjusted odds ratio $0.2 ; 95 \%$ CI $0.1-0.9 ; p=0.006)$. They concluded that the combination of oral antibiotics with a mechanical bowel preparation was the strongest predictor of decreased SSI.

A recent retrospective study, from Koeller et al. [9], involving 32.359 patients, from the American College of Surgeons National Survey Quality Improvement Program database, who underwent elective colorectal resections from 2012 to 2014 and divided into four groups (no MBP, MBP only, OA only and MBP + OA) have demonstrated (a) first, that the use of MBP alone before elective colorectal resection is ineffective to prevent SSI and should be abandoned; (b) second, in contrast, OA only and MBP + OA are associated with significantly decreased risks of SSI and are not associated with increased risks of other adverse outcomes compared with MBP or with no preparation. In multivariate analysis, odd ratios for MBP + OA ranged from 0.43 to 0.57 for SSI, leakage rate and death, suggesting a very important effect of the use of MBP plus OA. Which is also to be noted is that OA only also did better than MBP alone or no MBP with odd ratios ranging from 0.37 to 0.63 for the same criteria. This result suggested that maybe OA only is enough before colorectal surgery. Authors concluded that prospective studies to determine the efficacy of $\mathrm{OA}$ are required 
but that in the interim, MBP + OA should be used routinely before elective colorectal resection.

Although many randomized studies and meta-analyses have already suggested that the adjunction of OA to systemic antibiotics reduced the risk of SSI before colorectal surgery [10], to our knowledge, there is no RCT evaluating the respective benefit of OA and MBP before colonic and rectal surgery. For example, Hata et al. [11] published in 2016 a RCT comparing OA versus OA plus systemic antibiotics (SA) before colorectal surgery. They demonstrated that SSI was reduced in patients receiving $\mathrm{OA}+\mathrm{SA}$ versus SA only $(7.3 \%$ vs $12.8 \% ; p=0.028)$, but in this study, patients included presented either colonic or rectal cancer, which is confusing for the interpretation of the results. Furthermore, MBP was given in some patients but was not assessed in this study. Similarly, Ikeda et al. [12] published a RCT including also colonic and rectal surgery, with approximately $85 \%$ of patients undergoing MBP in both groups making interpretation of the possible benefit of both OA + MBP impossible.

Very recently, a RCT that focused only on patients with Crohn's disease [13] was also published: 335 patients scheduled to undergo intestinal resection with an open approach were randomly assigned to receive both preoperative OA and SA prophylaxis versus SA alone. The incidence of incisional SSI was significantly lower after OA + SA than after SA only: $7.4 \%$ vs $16.7 \%(p=0.01)$. However, all the patients received MBP and both small bowel and colorectal surgery were included in the same study.

One of the concerns using possibly OA in all the patients undergoing colorectal surgery is the potential risk of Clostridium difficile (CD) infection. Al-Mazrou et al. [14], evaluated the impact of OA on the development of $\mathrm{CD}$ infection in patients undergoing colectomy: $\mathrm{CD}$ occurs in $\approx 1$ to $7 \%$ of patients after colorectal surgery [15]. Comparing patients with OA and MBP to those who did not, Al-Mazrou et al. [14] showed that OA was associated with significantly lower postoperative $\mathrm{CD}$ infection and infectious complications, unplanned reoperations, mortality, length of stay and unplanned readmission. Also on multivariate analysis, OA reduced the odds for $\mathrm{CD}$ infection after colectomy. Additionally, in subgroup analysis of patients who did not develop any postoperative infectious complication, OA was associated with lower risk of $\mathrm{CD}$ infection.

These results confirm what has already been shown by Kim et al. [16] using a large observational cohort study data from the Michigan Surgical Quality CollaborativeColectomy Best Practices Project: they provided insight into the effect of bowel preparation on rates of CD infections in a study population of 2475 patients creating 957 paired cases $(n=1914)$ and comparing patients receiving MBP with OA with patients with no MBP; patients with $\mathrm{MBP}+\mathrm{OA}$ were actually less likely to develop postoperative $\mathrm{CD}$ infections than those who received no bowel preparation.
In conclusion, this risk of $\mathrm{CD}$ infection seems not to a real problem in receiving OA before colorectal surgery.

In conclusion, in 2019, there is a lot of evidence suggesting that $\mathrm{MBP}+\mathrm{OA}$ should be the new gold standard for colorectal surgery. But because randomized studies are still lacking, we do not know exactly if it is true for both colonic and rectal surgery. Furthermore, we still need to assess if only $\mathrm{OA}$ is enough or if both MBP and OA must be given to all the patients undergoing colorectal surgery. Nowadays, no randomized studies with homogeneous groups of patients are available in the literature; patients undergoing rectal cancer surgery must be separate from those undergoing colonic cancer surgery. That is why randomized trials with specific arms are needed [17].

In France, we have organized two randomized studies that started last year: one concerning patients undergoing surgery for colonic cancer (COLONPREP) and comparing four groups (none versus MBP versus OA alone versus both), and the another concerning rectal cancer patients (PREPACOL2) comparing two groups (MBP alone versus both $\mathrm{MBP}+\mathrm{OA}$ ). Before the results of these two studies, and the results of similar studies in progress worldwide, at least in France, we still continue to propose MBP for rectal cancer surgery but not for colonic cancer surgery.

\section{Compliance with ethical standards}

Conflict of interest The authors declare that they have no conflict of interest.

Research involving human participants and/or animals This article does not contain any studies with human participants or animals performed by any of the authors.

Informed consent For this type of study, formal consent is not required.

Funding No funding was obtained for this project.

\section{References}

1. Keighley M, Williams N (1997) Mechanical bowel preparation. Saunders, Philadelphia, pp 73-95

2. Guenaga KF, Matos D, Wille-Jørgense P (2011) Mechanical bowel preparation for elective colorectal surgery (review). Cochrane Collab 9:1-41

3. Cao F, Li J, Li F (2012) Mechanical bowel preparation for elective colorectal surgery: updated systematic review and meta-analysis. J Colorect Dis 27:803-810

4. Bretagnol F, Panis Y, Rullier E et al (2010) Rectal cancer surgery with or without bowel preparation. Ann Surg 252:863-868

5. Nichols RL, Broido P, Condon RE et al (1973) Effect of preoperative neomycin-erythromycin intestinal preparation on the incidence of infectious complications following colon surgery. Ann Surg 178:453-462 
6. Matheson DM, Arabi Y, Baxter-Smith D et al (1978) Randomized multicenter trial of oral bowel preparation and microbials for elective colorectal operations. BJS 65:597-600

7. Migaly J, Bafford AC, Francone TD et al (2019) The American Society of Colon and Rectal Surgeons Clinical Practice Guidelines for the use of bowel preparation in elective colon and rectal surgery. Dis Colon Rectum 62:3-8

8. Ohman KA, Wan L, Guthrie T et al (2017) Combination of oral antibiotics and mechanical bowel preparation reduces surgical site infection in colorectal surgery. J Am Coll Surg 225:465-471

9. Koller SE, Bauer KW, Egleston BL et al (2018) Comparative effectiveness and risks of bowel preparation before elective colorectal surgery. Ann Surg 267:734-742

10. Chen M, Song X, Chen LZ, Zhang XL (2016) Comparing mechanical bowel preparation with both oral and systemic antibiotics versus mechanical bowel preparation and systemic antibiotics alone for the prevention of surgical site infection after elective colorectal surgery: a meta-analysis of randomized controlled clinical trials. Dis Colon Rectum 59:70-78

11. Hata H, Yamaquchi T, Hasegawa S et al (2016) Oral and parenteral versus parenteral antibiotic prophylaxis in elective laparoscopic colorectal surgery (JMTO PREV 07-01): a phase 3, multicenter, open-label, randomized trial. Ann Surg 263:1085-1091

12. Ikeda A, Konishi T, Ueno M et al (2016) Randomized clinical trial of oral and intravenous versus intravenous antibiotic prophylaxis for laparoscopic colorectal resection. BJS 103:1608-1615
13. Uchino M, Ikeuchi H, Bando T (2019) Efficacy of preoperative oral antibiotic prophylaxis for the prevention of surgical site infections in patients with Crohn's disease a randomized controlled trial. Ann Surg 269:420-426

14. Al-Mazrou AM, Hyde LZ, Suradkar K et al (2018) Effect of inclusion or oral antibiotics with mechanical bowel preparation on the risk of Clostridium difficile infection after colectomy. J Gastrointest Surg 22:1968-1975

15. Krapohl GL, Morris AM, Cai S et al (2013) Preoperative risk factors for postoperative Clostridium difficile infection in colectomy patients. Am J Surg 205:343-347

16. Kim EK, Sheetz KH, Bonn J et al (2014) A statewide colectomy experience: the role of full bowel preparation in preventing surgical site infection. Ann Surg 259:310-314

17. Patchen Dellinger E (2015) Should a scheduled colorectal operation have a mechanical bowel prep, preoperative oral antibiotics, both, or neither? Ann Surg 261:1041-1043

Publisher's Note Springer Nature remains neutral with regard to jurisdictional claims in published maps and institutional affiliations. 